\title{
Origin of Low Carrier Mobilities in Halide Perovskites
}

\author{
Samuel Poncé, ${ }^{\dagger}$ Martin Schlipf, ${ }^{\dagger}$ and Feliciano Giustino*,†,‡ \\ $\dagger$ Department of Materials, University of Oxford, Parks Road, Oxford OX1 3PH, United \\ Kingdom \\ $\ddagger$ Department of Materials Science and Engineering, Cornell University, Ithaca, New York \\ 14853, USA \\ E-mail: feliciano.giustino@materials.ox.ac.uk
}

\begin{abstract}
Halide perovskites constitute a new class of semiconductors that hold promise for low-cost solar cells and optoelectronics. One key property of these materials is the electron mobility, which determines the average electron speed due to a driving electric field. Here we elucidate the atomic-scale mechanisms and theoretical limits of carrier mobilities in halide perovskites by performing a comparative analysis of the archetypal compound $\mathrm{CH}_{3} \mathrm{NH}_{3} \mathrm{PbI}_{3}$, its inorganic counterpart $\mathrm{CsPbI}_{3}$, and a classic semiconductor for LEDs, wurtzite GaN, using cutting-edge many-body ab initio calculations. We demonstrate that low-energy longitudinal-optical phonons associated with fluctuations of the $\mathrm{Pb}-\mathrm{I}$ bonds ultimately limit the mobility to $80 \mathrm{~cm}^{2} / \mathrm{Vs}$ at room temperature. By extending our analysis to a broad class of compounds, we identify a universal scaling law for the carrier mobility in halide perovskites, and we establish the design principles to realize high-mobility materials.
\end{abstract}




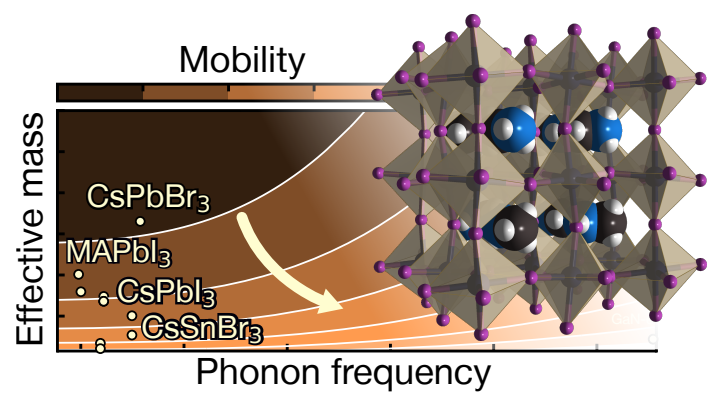

The discovery of halide perovskites has ushered in a new era in photovoltaics and optoelectronics research. ${ }^{1,2}$ Originally introduced as active layers for solar cells, ${ }^{3-5}$ organic-inorganic lead-halide perovskites and related compounds have shown great promise for a diverse array of applications, including photodetectors, light-emitting diodes, semiconductor lasers, optical refrigerators, X-ray and gamma-ray detectors, photocatalysts, thermochromic windows, metal-halide perovskite transitors, and thin-film field-effect transistors. ${ }^{6-13}$ The main appeal of these materials is that they can be fabricated via solution process, yet they exhibit outstanding optoelectronic properties, to the point that they have been designated as the 'first high-quality halide semiconductors'. ${ }^{14}$ In all these devices the carrier mobility plays a central role. For example, it is a key ingredient of optoelectronic device simulators based on drift-diffusion models. Furthermore, the photoconductive gain in photodetectors and the cutoff frequency in thin-film transistors are directly proportional to the mobility. The cutoff frequency is especially important for realizing $\mathrm{GHz}$ wireless connectivity. ${ }^{15}$

Further progress in the use of halide perovskites in electronics, optoelectronics, and energy will require us to understand and control the microscopic mechanisms that underpin the optical and transport properties of these materials. While a broad consensus has emerged on their optical properties, ${ }^{16}$ understanding carrier transport is proving elusive. ${ }^{17}$ For example, measurements of the carrier mobility show striking variations among different labs, ranging from $0.07 \mathrm{~cm}^{2} / \mathrm{Vs}$ for room-temperature electron drift mobilities measured in thin films of $\mathrm{CH}_{3} \mathrm{NH}_{3} \mathrm{PbI}_{3}$ prepared via spin-coating, ${ }^{13}$ to $67 \mathrm{~cm}^{2} / \mathrm{Vs}$ extracted from current-voltage measurements on single-crystals of $\mathrm{CH}_{3} \mathrm{NH}_{3} \mathrm{PbI}_{3} \cdot{ }^{18}$ Such a large variability in the reported carrier mobilities has been ascribed to differences in sample morphology (single crystals vs. 
films), preparation routes (solid state vs. solution or evaporation), and measurements techniques (e.g. Hall effect, time-of-flight, THz conductivity, photoluminescence quenching). ${ }^{17}$ In the face of this high level of uncertainty in the measurements, it is important to identify the ultimate theoretically-achievable carrier mobility, and which materials parameters control this limit, so as to set out clear guidelines for materials and device engineering.

In this work we turn to quantum-mechanical calculations in order to establish the ultimate mobility of the archetypal organic-inorganic perovskite, methylammonium lead triiodide $\mathrm{CH}_{3} \mathrm{NH}_{3} \mathrm{PbI}_{3}\left(\mathrm{MAPbI}_{3}\right)$. There have been several attempts in this direction, but since earlier calculations relied on empirical scattering rates ${ }^{19,20}$ or deformation potentials, ${ }^{21,22}$ these estimates carry the very same uncertainties that we seek to eliminate. At variance with earlier work, we employ a recently-developed ab initio many-body computational technique which enables predictive, parameter-free, and accurate calculations of mobilities, with deviations from the measurements as little as a few percent. ${ }^{23}$ This new tool allows us to establish which atomic-scale mechanism dominates the mobility in halide perovskites, and answer the question on why the mobility of $\mathrm{MAPbI}_{3}$ is orders of magnitude lower than in inorganic semiconductors such as GaN. Using this understanding we propose strategies to overcome the mobility bottleneck and illustrate the practical benefits of realizing highmobility perovskites.

Figure 1(a) shows the phonon-limited mobility of $\mathrm{MAPbI}_{3}$ calculated for a perfect, defectfree bulk crystal in the low-temperature orthorhombic Pnma structure. In this structure the $\mathrm{MA}^{+}$cations (methylammonium, $\mathrm{CH}_{3} \mathrm{NH}_{3}^{+}$) have definite crystallographic orientations, as shown in the ball-stick model of Figure 2(a). This model is accurate for the orthorhombic phase of $\mathrm{MAPbI}_{3}(\mathrm{~T}=0-165 \mathrm{~K})$, and provides a good approximation to the tetragonal $\mathrm{I} / \mathrm{mcm}$ phase $(\mathrm{T}=165-327 \mathrm{~K})$, where the $\mathrm{MA}^{+}$cation spins around the $\mathrm{C}-\mathrm{N}$ axis and aligns along one of four preferred orientations in the $a b$ plane. ${ }^{33-35}$ For the high-temperature $\operatorname{Pr} \overline{3} m$ cubic phase $(T>327 \mathrm{~K})$, neutron scattering indicates that the organic cations are rotationally-disordered, ${ }^{36}$ with preferential orientation along the [111], [100], and [011] 

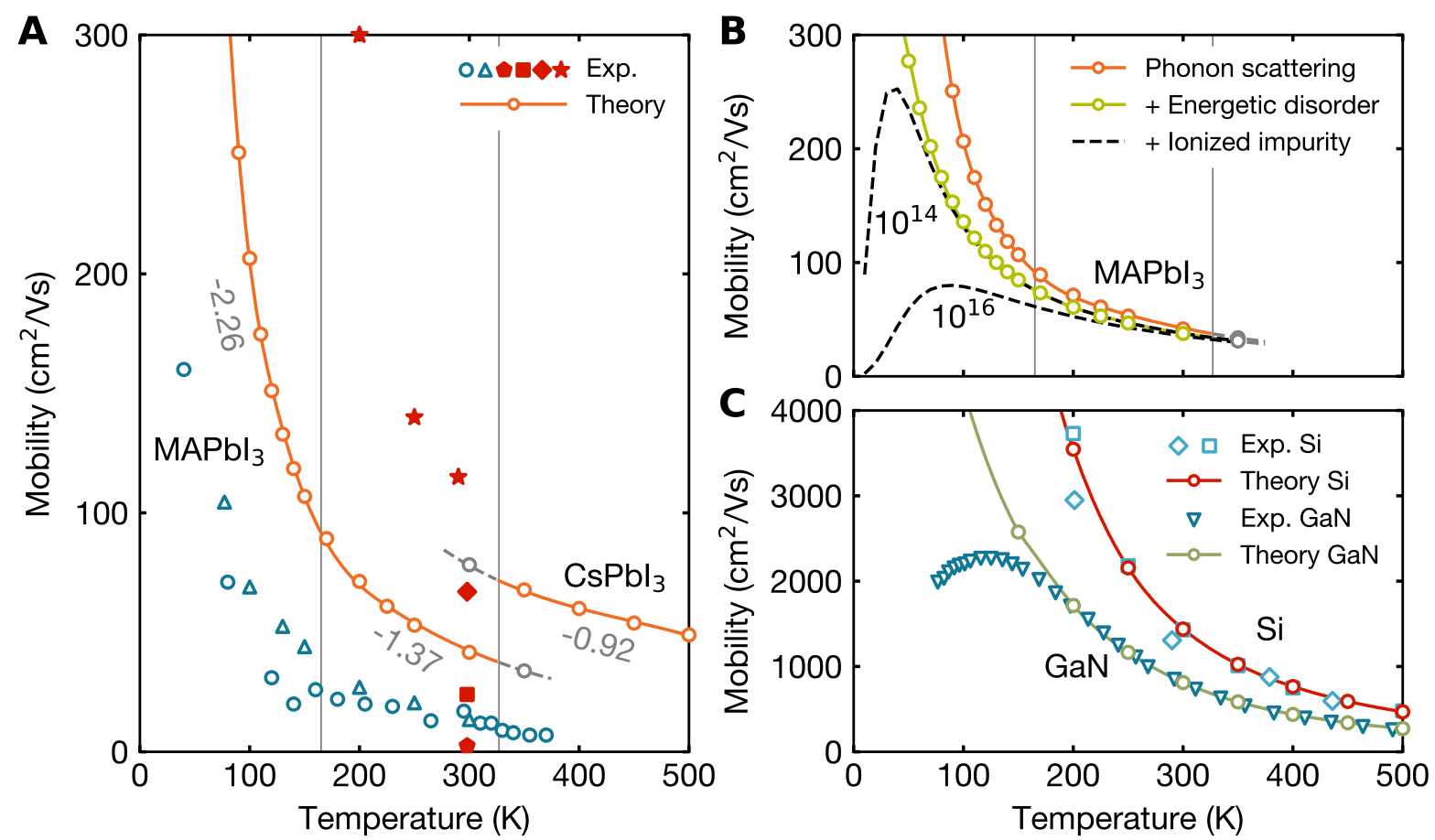

Figure 1: Temperature-dependent carrier mobilities in $\mathrm{MAPbI}_{3}, \mathrm{CsPbI}_{3}$, and GaN. (A) Average electron and hole phonon-limited mobilities calculated from the $a b i n i-$ tio many-body Boltzmann equation for $\mathrm{MAPbI}_{3}$ in the Pnma structure (circles and solid orange lines between $0-350 \mathrm{~K}$ ) and $\mathrm{CsPbI}_{3}$ in the $P m \overline{3} m$ structure, at the lattice parameter of $\mathrm{MAPbI}_{3}$, between $250-500 \mathrm{~K}$. The experimental data are from Ref. ${ }^{24}$ (circles, optical pump-THz probe) and Ref. ${ }^{25}$ (triangles, ultra-broadband $\mathrm{THz}$ photoconductivity) taken on thin films, whereas the filled red symbols are taken on single crystals in Ref. ${ }^{26}$ (pentagon, space-charge-limited current), Ref. ${ }^{27}$ (square, time of flight), Ref. ${ }^{18}$ (diamond, dark currentvoltage) and Ref. ${ }^{28}$ (star, time of flight). All calculations were performed without any artificial broadening, and therefore represent theoretical upper bounds. The vertical bars indicate the boundaries of the orthorhombic, tetragonal, and cubic phases of $\mathrm{MAPbI}_{3}$ while the gray numbers indicate the exponent for the temperature dependence of the three phases. (B) Impact of energetic disorder and ionized impurity scattering on the ideal mobility of $\mathrm{MAPbI}_{3}$ : we compare the average of the phonon-limited electron and hole mobilities from panel (A) (orange line) with that obtained after including a $13 \mathrm{meV}$ broadening of the electronic states (from the Urbach tail measured in Ref. ${ }^{29}$ )(green line), and scattering by ionized impurities at the concentrations $10^{14}$ and $10^{16} \mathrm{~cm}^{-3}$, from Ref. ${ }^{30}$ (black dashed lines). The impurity scattering is calculated using the semi-empirical Brook-Herring model.(C) Electron mobility of GaN as a function of temperature: present calculations (green circles and line) vs. mobilities extracted from Hall effect measurements in Ref. ${ }^{31}$ (triangles). The theoretical electron mobility of silicon is from Ref. ${ }^{23}$ (red circles and line) and the experiments (diamonds and squares) are from Ref. ${ }^{32}$ 
directions. In order to mimick orientational disorder we perform calculations for cubic $\mathrm{CsPbI}_{3}$ at the lattice parameter of $\mathrm{MAPbI}_{3}$, as shown by the ball-stick model in Figure 2(b). The corresponding mobilities are reported in Fig. 1(a) in the temperature range above $327 \mathrm{~K}$.

In Fig. 1(a) we see that the calculated electron and hole mobilities decrease with temperature, from $\sim 500 \mathrm{~cm}^{2} / \mathrm{Vs}$ below $100 \mathrm{~K}$ to $28 \mathrm{~cm}^{2} / \mathrm{Vs}$ and $47 \mathrm{~cm}^{2} / \mathrm{Vs}$ at room temperature for electrons and holes, respectively. These values constitute theoretical upper bounds, since at this stage we are only considering electron-phonon scattering in the undoped, defectfree crystal. Since our data correspond to intrinsic mobilities in bulk perovskites, the most appropriate way to compare with experiments is to consider corrected Hall measurements, time-of-flight or steady-state photoconductivity measurements ${ }^{28,37,38}$ on $\mathrm{MAPbI}_{3}[\mathrm{red}$ symbols in Fig. 1(a)]. To extend the comparison throughout the entire temperature range, we also consider transient $\mathrm{THz}$ data on thin films. When compared to $\mathrm{THz}$ photoconductivity data on thin films ${ }^{24,25}$ we see that our calculations yield higher values, as expected, but a similar temperature dependence. In fact, by fitting the calculated data in the temperature ranges corresponding to the orthorhombic, tetragonal, and cubic phases using a power law $\left(a T^{b}\right)$, we find that the mobility scales as $\mu \sim T^{-2.26}, \mu \sim T^{-1.37}$, and $\mu \sim T^{-0.92}$, respectively. The exponents determined here broadly agree with previous theoretical work based on analytical models. ${ }^{20,39,40}$ In addition, our calculated temperature exponent in the tetragonal phase agrees with the value of $-3 / 2$ obtained in the experiments. ${ }^{24}$ This power law was previously attributed to acoustic-phonon scattering, ${ }^{25,37}$ but as we will discuss below the dominant scattering mechanism is from polar phonons, in agreement with recent analytical model calculations. ${ }^{20,41}$ At the tetragonal-to-cubic transition temperature $(327 \mathrm{~K})$ the mobilities of the cubic $\mathrm{CsPbI}_{3}$ phase reach values of $68 \mathrm{~cm}^{2} / \mathrm{Vs}$ and $76 \mathrm{~cm}^{2} / \mathrm{Vs}$ for electrons and holes, respectively. The increase with respect to the orthorhombic $\mathrm{MAPbI}_{3}$ structure is due to stiffer optical phonons and smaller effective masses, as we show below. In Fig. 1(b) we can see that scattering by charged impurities in $\mathrm{MAPbI}_{3}$ provides a negligible contribution to the mobility at temperatures above $150 \mathrm{~K}$; therefore, in the temperature range relevant 

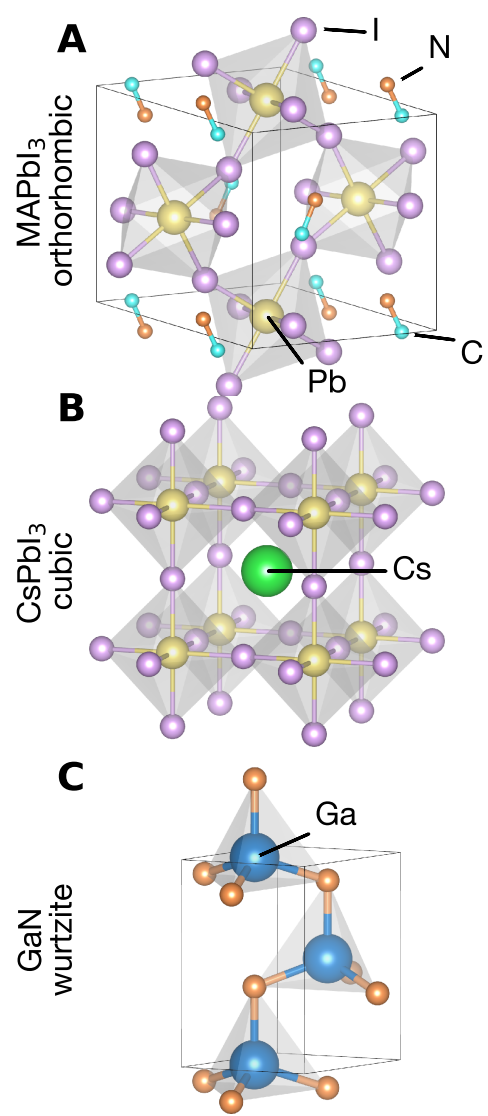

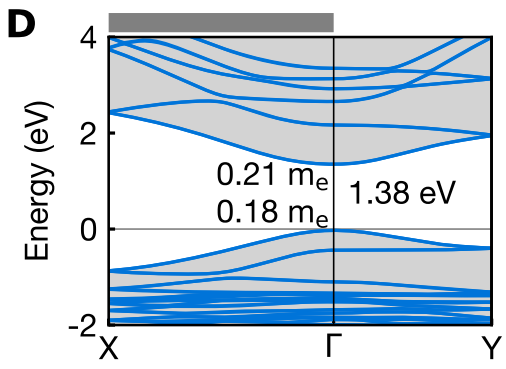

E
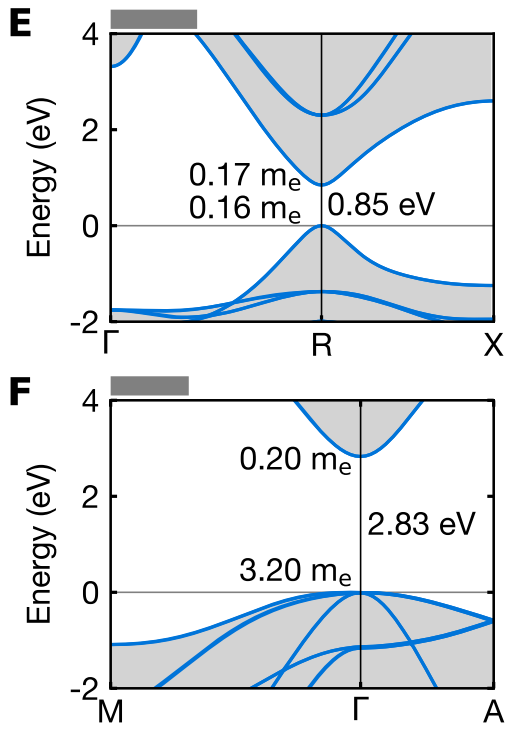
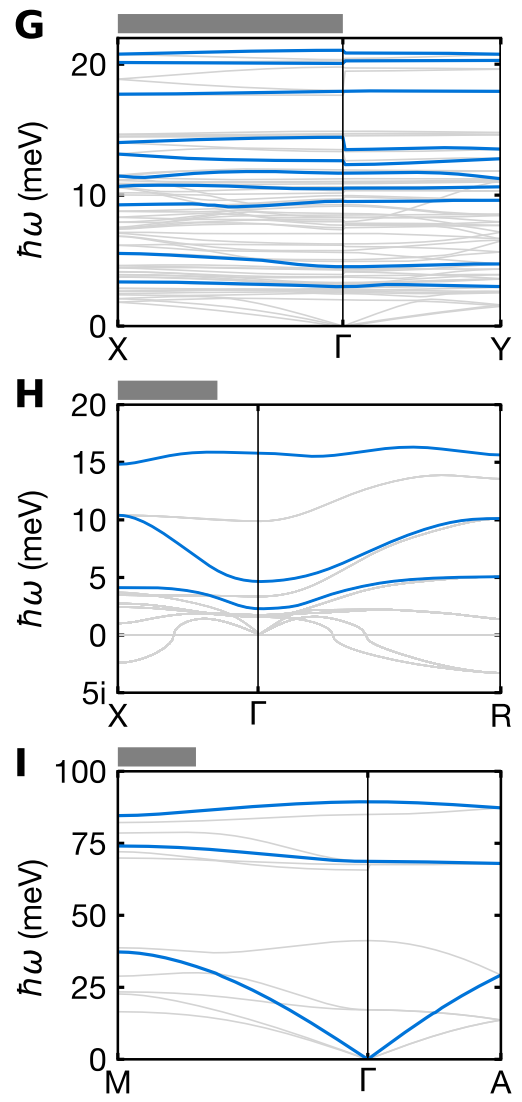

Figure 2: Crystal structure, band structure, and phonon dispersion relations of $\mathrm{MAPbI}_{3}, \mathrm{CsPbI}_{3}$, and GaN. (A-C) Ball-stick models of $\mathrm{MAPbI}_{3}$ in the orthorhombic Pnma structure, $\mathrm{CsPbI}_{3}$ in the cubic $P m \overline{3} m$ structure, and wurtzite GaN, respectively. For the $\mathrm{MA}^{+}$cations only the $\mathrm{C}$ and $\mathrm{N}$ atoms are shown for clarity. (D-F) Calculated GW band structures of $\mathrm{MAPbI}_{3}, \mathrm{CsPbI}_{3}$, and $\mathrm{GaN}$, for the models shown in (A)-(C). The valence and conduction bands are shaded for clarity, and the band gaps and effective masses are indicated. (G) Calculated phonon dispersion relations of $\mathrm{MAPbI}_{3}$ for the model in $(\mathrm{A})$. The lines highlighted in blue represent the LO phonons with strongest electron-phonon coupling. (H) Phonon dispersion relations of $\mathrm{CsPbI}_{3}$, using the same color code as in (D). The soft modes around the high-symmetry point $R=1 / 2(1,1,1)$ (in reciprocal lattice units) do not contribute to the mobility, as shown in Supporting Information, section 1. (I) Phonon dispersion relations of wurtzite GaN. In this case we highlight in blue the LO phonons around $89 \mathrm{meV}$, and the acoustic phonons leading to piezoacoustic scattering. We note that the scales of the horizontal axes in D-F and G-I differ; for clarity we indicate in each panel the reciprocal lattice length $\Gamma$-X of orthorhombic $\mathrm{MAPbI}_{3}$ as gray bar. 
for device operation, carrier transport in this system is predominantly phonon-limited.

In order to validate our present calculations we also show in Fig. 1(c) the calculated electron mobilities of prototypical semiconductors for photovoltaics and LEDs: silicon and wurtzite GaN. The latter exhibits an electron effective mass close to that of $\mathrm{MAPbI}_{3}$. Here we see that the agreement with experiments is excellent for temperatures above $150 \mathrm{~K} .{ }^{31}$ The deviation between our calculations for GaN and experiments at lower temperatures relates to scattering by crystal defects, which we have not included. Overall this comparison for silicon and gallium nitride demonstrates that our methodology is capable of delivering highly accurate mobilities without using any empirical parameters. Based on our calculated values for the orthorhombic and cubic phases in Fig. 1(a), we predict that the ultimate average electron/hole mobility of $\mathrm{MAPbI}_{3}$ at room temperature lies between $30 \mathrm{~cm}^{2} / \mathrm{Vs}$ and $80 \mathrm{~cm}^{2} /$ vs. Interestingly, the highest mobility measured on $\mathrm{MAPbI}_{3}$ single crystals, $67 \mathrm{~cm}^{2} / \mathrm{Vs}^{18}$ is already approaching this theoretical limit.

We now proceed to a detailed analysis of the mechanisms underpinning the mobilities in Fig. 1(a). The calculations leading to these data were performed within the framework of the $a b$ initio Boltzmann-transport formalism, using the self-energy relaxation time approximation. ${ }^{23}$ The electron mobility tensor reads:

$$
\mu_{\mathrm{e}, \alpha \beta}=-\frac{e}{n_{\mathrm{e}} \Omega} \sum_{n \in \mathrm{CB}} \int \frac{d \mathbf{k}}{\Omega_{\mathrm{BZ}}} \frac{\partial f_{n \mathbf{k}}}{\partial \varepsilon_{n \mathbf{k}}} v_{n \mathbf{k}, \alpha} v_{n \mathbf{k}, \beta} \tau_{n \mathbf{k}},
$$

where $e$ is the electron charge, $n_{\mathrm{e}}$ is the electron density, $\Omega$ and $\Omega_{\mathrm{BZ}}$ are the volume of the crystalline unit cell and the first Brillouin zone, respectively. The sum is restricted to states in the conduction bands (CB) with energy $\varepsilon_{n \mathbf{k}}$, band index $n$ and wavevector $\mathbf{k} . f_{n \mathbf{k}}$ is the Fermi-Dirac function, and $v_{n \mathbf{k}, \alpha}=\hbar^{-1} \partial \varepsilon_{n \mathbf{k}} / \partial k_{\alpha}$ is the electron band velocity along the Cartesian direction $\alpha$ ( $\hbar$ is the reduced Planck constant). The relaxation time in Eq. (1) is 
given by: ${ }^{42}$

$$
\frac{1}{\tau_{n \mathbf{k}}}=\frac{2 \pi}{\hbar} \sum_{m \nu \sigma} \int \frac{d \mathbf{q}}{\Omega_{\mathrm{BZ}}}\left|g_{m n \nu}(\mathbf{k}, \mathbf{q})\right|^{2}\left[(1+\sigma) / 2-\sigma f_{m \mathbf{k}+\mathbf{q}}+n_{\mathbf{q} \nu}\right] \delta\left(\varepsilon_{n \mathbf{k}}-\varepsilon_{m \mathbf{k}+\mathbf{q}}-\sigma \hbar \omega_{\mathbf{q} \nu}\right)
$$

Here $g_{m n \nu}(\mathbf{k}, \mathbf{q})$ is the matrix element for the scattering of an electron from the initial state $|n \mathbf{k}\rangle$ to the final state $|m \mathbf{k}+\mathbf{q}\rangle$ via a phonon of branch $\nu$, wavevector $\mathbf{q}$, and frequency $\omega_{\mathbf{q} \nu}$. $n_{\mathbf{q} \nu}$ is the Bose-Einstein occupation of this phonon, and $\sigma$ takes the values \pm 1 for phonon emission/absorption, respectively. The temperature enters Eqs. (1) and (2) via the FermiDirac and Bose-Einstein distributions. The mobility tensor for holes is obtained analogously, by restricting the summation in Eq. (1) over the valence bands.

All the quantities entering Eqs. (1) and (2) are calculated entirely from first principles. In particular the band structures include state-of-the-art GW quasiparticle corrections to density-functional theory with spin-orbit coupling; the phonons are obtained from densityfunctional perturbation theory (DFPT); and the electron-phonon matrix elements are evaluated from DFPT and ab initio Wannier interpolation, as described in the Methods section. The scattering of electrons by all phonons, namely acoustic, optical, transverse, and longitudinal modes is included without approximations. To capture both long-range and short-range electron-phonon scattering with the same accuracy, the integrals are evaluated using ultradense Brillouin zone grids. ${ }^{23}$ In principle we could go one step beyond Eq. (1) and evaluate mobilities using the self-consistent Boltzmann equation. However, in systems with cubic symmetry at low fields, this usually leads to a modification of the relaxation-time results by $5-10 \%,{ }^{23}$ therefore we do not pursue this direction. In a recent paper, ${ }^{41}$ we established using Feynman's model ${ }^{43}$ that the electron-phonon coupling in $\mathrm{MAPbI}_{3}$ leads to the formation of large polarons with a radius between 62 and $81 \AA$, extending over more than $20 \mathrm{PbI}_{6}$ octahedra. We therefore expect that these polarons should not have a strong effect on the scattering matrix elements, and neglect their effect on mobility. Work on the impact of large polarons on the mobility of perovskites is ongoing ${ }^{40,44-47}$ but remains an open question. 
Figure 2 shows ball-stick models of $\mathrm{MAPbI}_{3}, \mathrm{CsPbI}_{3}$, and $\mathrm{GaN}$, and compares the respective GW band structures and phonon dispersion relations. $\mathrm{MAPbI}_{3}$ and $\mathrm{CsPbI}_{3}$ exhibit similar effective masses for electrons and holes: $m_{\mathrm{e}}^{*} / m_{\mathrm{h}}^{*}=0.18 / 0.21 m_{\mathrm{e}}$ vs. $m_{\mathrm{e}}^{*} / m_{\mathrm{h}}^{*}=0.16 / 0.17 m_{\mathrm{e}}$, respectively ( $m_{\mathrm{e}}$ is the free electron mass). From these values we obtain a reduced effective mass in $\mathrm{MAPbI}_{3}$ of $0.098 m_{\mathrm{e}}$, in close agreement with magnetotransport measurements yielding $0.104 \pm 0.003 m_{\mathrm{e}} \cdot{ }^{48}$ The band gap of $\mathrm{MAPbI}_{3}$ is also in good agreement with experiments $\left(1.38 \mathrm{eV}\right.$ vs. $\left.1.6 \mathrm{eV}^{16}\right)$, the deviation of $0.2 \mathrm{eV}$ being in the typical range of $\mathrm{GW}$ calculations (Supporting Information, section 3). In our calculations we do not include temperature renormalization of the band gap since this effect is very small in $\mathrm{MAPbI}_{3}{ }^{16,49}$ and the effective mass does not change appreciably with temperature. ${ }^{41}$ In Fig. 2(e) we see that the band gap of $\mathrm{CsPbI}_{3}$ is considerably narrower than in $\mathrm{MAPbI}_{3}$. This effect relates to the absence of octahedral tilts in the cubic structure, as already discussed in previous work. ${ }^{50}$ Moving to GaN, in Fig. 2(f) we see that despite the much wider band gap, the electron effective mass is very similar to $\mathrm{MAPbI}_{3}\left(0.20 m_{\mathrm{e}}\right)$, therefore it is appropriate to use GaN as a benchmark. Additional data on the effective masses are reported in Supporting Information, Table S2 for completeness. From the phonon dispersion relations in Fig. 2(g-i) we see that $\mathrm{MAPbI}_{3}$ exhibits more branches that $\mathrm{CsPbI}_{3}$ and $\mathrm{GaN}$; this is simply related to the larger unit cell (48 atoms per cell as compared to 5 and 4 atoms/cell, respectively). In both $\mathrm{MAPbI}_{3}$ and $\mathrm{CsPbI}_{3}$ the vibrational modes of the $\mathrm{PbI}_{3}$ network extend up to $\sim 15 \mathrm{meV}$. The additional branches that can be seen for $\mathrm{MAPbI}_{3}$ in Fig. $2(\mathrm{~g})$ around $\sim 20 \mathrm{meV}$ are associated with librations of the $\mathrm{MA}^{+}$cations; these modes do not have a counterpart in $\mathrm{CsPb}_{3}$. The soft modes that are seen for $\mathrm{CsPbI}_{3}$ in Fig. 2(h) correspond to octahedral tilts, and are stabilized by anharmonic effects. ${ }^{51}$ Since they have no effect on the carrier mobilities (Supporting Information, section 1), we do not consider them when evaluating Eq. (2). In the case of GaN we see that the highest optical modes reach up to $\sim 90 \mathrm{meV}$, as a result of the much lighter masses of Ga and $\mathrm{N}$ as compared to $\mathrm{Pb}$ and $\mathrm{I}$.

We are now in a position to analyze the origin of carrier mobilities in $\mathrm{MAPbI}_{3}$. Fig- 

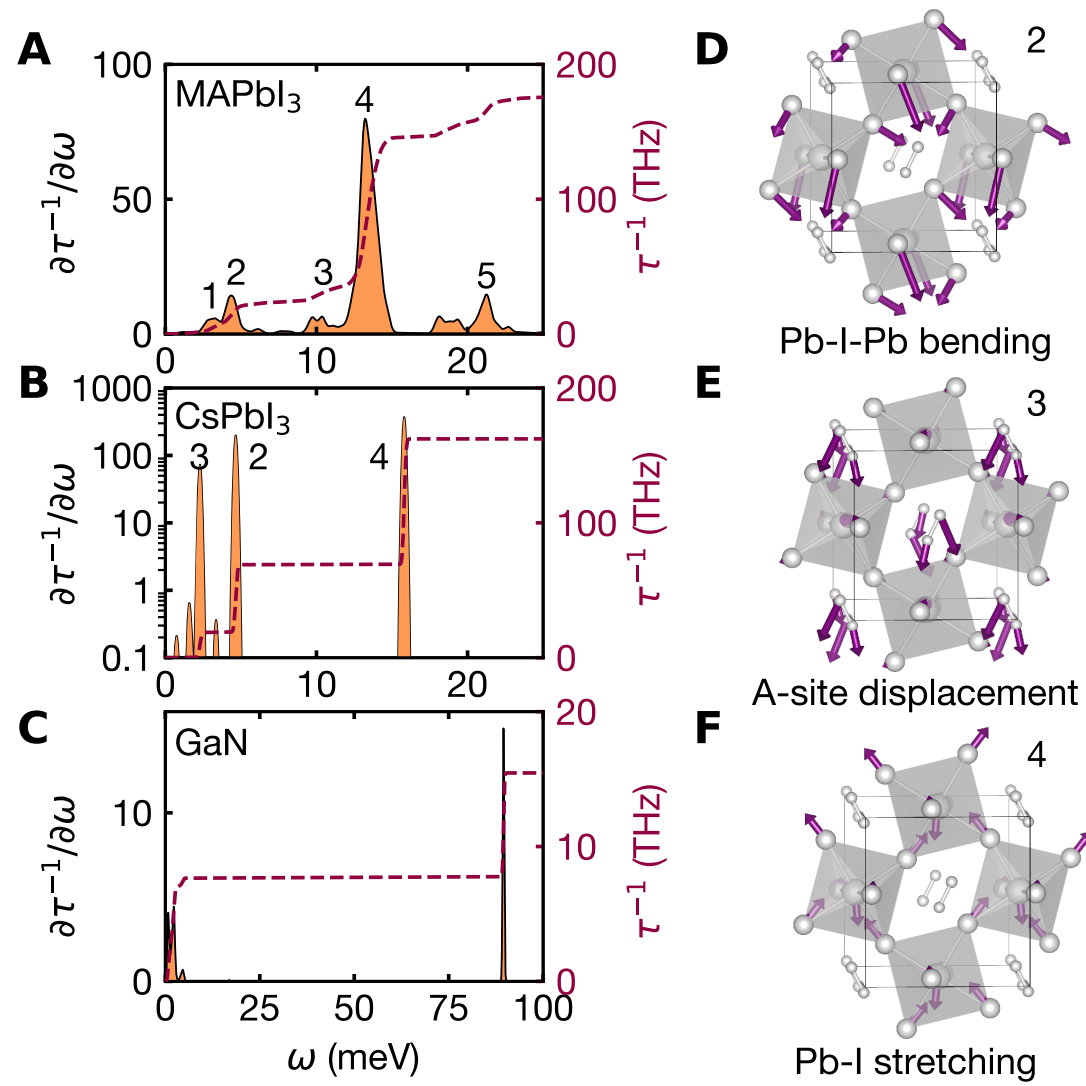

E
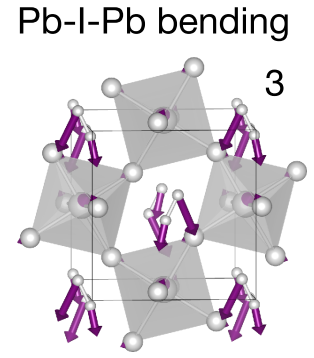

$\mathbf{F}$

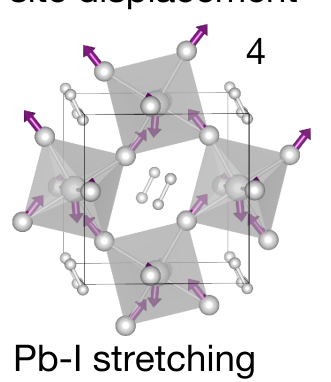

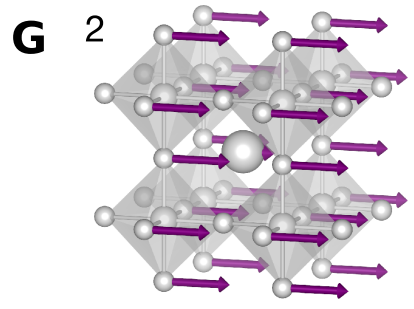

$\mathrm{Pb}-\mathrm{I}-\mathrm{Pb}$ bending

H

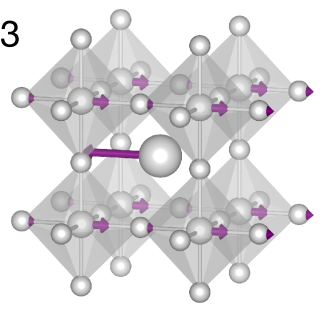

A-site displacement

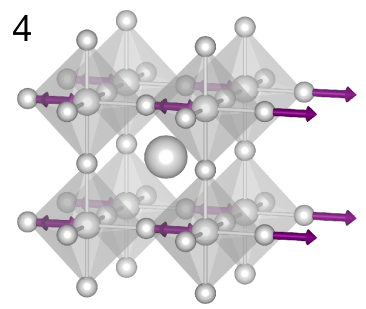

$\mathrm{Pb}-\mathrm{I}$ stretching

Figure 3: Electron-phonon scattering mechanisms in $\mathrm{MAPbI}_{3}, \mathrm{CsPbI}_{3}$, and $\mathrm{GaN}$. (A-C) Spectral decomposition of the contribution of each phonon with energy $\hbar \omega$ to the electron scattering rate $1 / \tau_{n \mathbf{k}}$, for $\mathrm{MAPbI}_{3}(\mathrm{~A}), \mathrm{CsPbI}_{3}(\mathrm{~B})$, and GaN $(\mathrm{C})$. The scattering rates are evaluated for electrons at an energy $3 / 2 k_{\mathrm{B}} T$ from the conduction band bottom, with $T=300 \mathrm{~K}$ (see Supporting Information, section 2). The height of the peaks indicates the contribution of each phonon (left vertical axis), and the integral $\int\left(\partial \tau^{-1} / \partial \omega\right) d \omega$ yields the total scattering rate (right vertical axis). The dashed line indicates the cumulative integral of the differential scattering rate. Acoustic scattering is negligible in $\mathrm{MAPbI}_{3}$ and $\mathrm{CsPbI}_{3}$, while the dominant contributions are from vibrations corresponding to LO phonons, as indicated by the peaks labeled 1-5 described in the main text and illustrated in (D-F). In the case of $\mathrm{GaN}$ approximately half of the scattering rate arises from piezoacoustic scattering, as shown by the peaks between $0-5 \mathrm{meV}$ in $(\mathrm{C})$. (D-F) Schematic representations of the atomic displacements of $\mathrm{MAPbI}_{3}$ corresponding to the peaks 2-4 identified in (A). (G-I) Same as in (D-F) but for $\mathrm{CsPbI}_{3}$.

ure $3(\mathrm{a}-\mathrm{c})$ show the contribution of each vibrational mode to the total scattering rate $1 / \tau_{n \mathbf{k}}$ in Eq. (2). The calculation of mobility in Eq. (1) requires to integrate over many electronic states, however for the present analysis we can focus on the most representative scattering rate (Supporting Information, section 2), which corresponds to states at an energy $3 / 2 k_{\mathrm{B}} T$ 
from the band edge, with $T=300 \mathrm{~K}$ ( $k_{\mathrm{B}}$ is the Boltzmann constant). Let us start from GaN and work our way back to $\mathrm{MAPbI}_{3}$. In Fig. 3(c) we see that in GaN the scattering rate is dominated by piezo-acoustic coupling with phonons around $3 \mathrm{meV}$ and by longitudinaloptical (LO) phonons around $89 \mathrm{meV}$; these modes are shown by blue lines in the phonon dispersion plots of Fig. 2(i). Moving to $\mathrm{CsPbI}_{3}$ in Fig. 3(b), we see three distinct peaks contributing to the scattering rate (labeled as $2,3,4$ ), as well as a small background contribution. The three sharp peaks originate from $\mathrm{LO}$ modes of $\mathrm{CsPbI}_{3}$, namely the bending mode at $2.3 \mathrm{meV}$ (peak 3), the A-site displacement mode at $4.6 \mathrm{meV}$ (peak 2), and the Pb-I stretch mode at $15.8 \mathrm{meV}$ (peak 4). The atomic displacements corresponding to these modes are shown in Fig. 3(g-i). The background is due to acoustic modes and transverse-optical (TO) phonons, but this contribution is much weaker than in GaN, since piezoacoustic scattering in $\mathrm{CsPbI}_{3}$ is forbidden by inversion symmetry. Of the three peaks, the $\mathrm{Pb}-\mathrm{I}$ stretch mode dominates, and accounts for approximately half of the scattering rate. Moving now to $\mathrm{MAPbI}_{3}$ in Fig. 3(a), we recognize distinct peaks around $4.3 \mathrm{meV}$ and $14.4 \mathrm{meV}$ (peaks 2 and 4), which are similar to the case of $\mathrm{CsPbI}_{3}$, only much broader. The broadening results from the hybridization of the $\mathrm{PbI}_{3}$ vibrations with those of the organic cations, ${ }^{52}$ although the character of the modes remains essentially unchanged. Around $3 \mathrm{meV}$ we find a weak $\mathrm{Pb}-\mathrm{I}-\mathrm{Pb}$ rocking mode (peak 1), and the A-site displacement mode is now at higher energy, at $10.2 \mathrm{meV}$ (peak 3). We also see a broad structure around $21 \mathrm{meV}$, which originates from librational modes of $\mathrm{MA}^{+}$(peak 5). The characteristic displacement patterns of three of those five modes are shown in Fig. 3(d-f). Our Boltzmann transport formalism relies on the assumption of harmonic phonons. The use of the harmonic approximation is justified, because the only anharmonic modes in the orthorhombic phase of $\mathrm{MAPbI}_{3}$ are associated with the spinning of the MA cation around the C-N axis with an energy of $16 \mathrm{meV}^{53}$ and, as shown in Fig. 3(a), these modes have a negligible contribution to the scattering rate. Also in the case of $\mathrm{MAPbI}_{3}$ the contribution of acoustic and TO phonons is essentially negligible, and piezoacoustic scattering is inactive. 
Using this understanding we can explain why the mobilities in halide perovskites are more than an order of magnitude smaller than in $n$-type GaN $\left(30-80 \mathrm{~cm}^{2} / \mathrm{Vs}\right.$ vs. $810 \mathrm{~cm}^{2} / \mathrm{Vs}$ at room temperature, see Fig. 1). Since we established that in $\mathrm{MAPbI}_{3}$ the carrier mobility is dominated by polar-phonon scattering, we can simplify Eqs. (1) and (2) to focus specifically on the contributions arising from Fröhlich interactions. We already noted that the effective masses of $\mathrm{MAPbI}_{3}$ are similar to the electron effective mass of $\mathrm{GaN}$, therefore the possible origin of the difference between $\mathrm{GaN}$ and $\mathrm{MAPbI}_{3}$ must reside in the scattering rates $1 / \tau_{n \mathbf{k}}$ in Eq. (2). A straightforward estimate shows that in GaN there are essentially no LO phonons at room temperature $\left(n_{\mathbf{q} \nu}=0.03\right.$ for $\hbar \omega_{\mathrm{LO}}=90 \mathrm{meV}$ and $\left.k_{\mathrm{B}} T=25.7 \mathrm{meV}\right)$, therefore scattering via LO phonon absorption is extremely weak. On the other hand, electrons within an energy $\hbar \omega_{\mathrm{LO}}$ cannot emit phonons otherwise the final states would be inside the band gap. Therefore, the electronic states that dominate the scattering integral due to the Fermi factor $\partial f_{n \mathbf{k}} / \partial \varepsilon_{n \mathbf{k}} \propto \exp \left(-\varepsilon_{n \mathbf{k}} / k_{\mathrm{B}} T\right)$ in Eq. (1) are essentially immune to LO-phonon scattering. This effect accounts for the large electron mobilities observed in GaN. These considerations cease to hold in the case of $\mathrm{MAPbI}_{3}$. In fact we now have LO phonons in the range $\hbar \omega_{\mathrm{LO}}=5-20 \mathrm{meV}$. As a result, both phonon absorption and emission are allowed, and the corresponding Bose-Einstein factors are large $\left(n_{\mathbf{q} \nu}=0.8-4.6\right)$. The electron scattering rates are correspondingly much higher than in GaN, as seen in Fig. 2(a) and (c). Therefore the large difference in mobility between $\mathrm{MAPbI}_{3}$ and $\mathrm{GaN}$ originates primarily from different phonon energy scales.

In order to place this reasoning on more quantitative grounds we developed a simplified conceptual model, obtained from Eqs. (1) and (2) by considering electrons with effective mass $m^{*}$ interacting with a single LO phonon of energy $\hbar \omega_{\mathrm{LO}}$ via the standard Fröhlich coupling strength $\alpha$. In Supporting Information, section 2 we show that, under these approximations, the mobility depends only on two dimensionless parameters, a 'polar mass' $\alpha\left(m^{*} / m_{\mathrm{e}}\right)$ and a 'reduced frequency' $\hbar \omega_{\mathrm{LO}} / k_{\mathrm{B}} T$. Figure 4 shows a map of the mobility as a function of these parameters. We see that high mobilities can be achieved when the polar mass is small and 
the phonon frequency is large. The complete analytical expression of the mobility in terms of these parameters is given in Supporting Information, section 2 [see Eqs. (S6)-(S9)], however to a first approximation this trend can be captured by the dimensionless scaling law:

$$
\mu /\left(e \hbar / m_{\mathrm{e}} k_{\mathrm{B}} T\right)=\left[0.052\left(\hbar \omega_{\mathrm{LO}} / k_{\mathrm{B}} T\right)^{3.3}+0.34\right] /\left(\alpha m^{*} / m_{\mathrm{e}}\right) .
$$

We called this relation a "universal scaling law" to highlight its broad validity across the class of halide perovskites considered here, and its dependence on dimensionless scaling parameters. Using this law, a stiffening of the phonon frequencies by a factor of $89 / 13 \simeq$ $6.8\left(\mathrm{GaN} / \mathrm{MAPbI}_{3}\right)$ leads to an increase in the room-temperature mobility by a factor of $\simeq$ 9.7. Furthermore, a reduction of the polar mass by $0.34 / 0.09 \simeq 3.8$ (Supporting Information, Table S2) increases the mobility by the same factor. These two effects combined fully explain why the mobility attainable in $\mathrm{MAPbI}_{3}$ is $\sim 40$ times lower than in a classic optoelectronic material like GaN. We can estimate the accuracy of our calculations by noting that our computed reduced effective mass underestimates the experimental value by $6 \%$, and our LO phonon frequency (see Table S3) overestimates the experimental value by $10 \% .{ }^{54}$ These two effects tend to cancel according to Eq. (3), and lead to an uncertainty of $6 \%$ in the mobility.

Guided by the universal mobility map in Fig. 4(a), we explored the possibility of tuning mobilities in halide perovskites by anion and cation substitution. Naively one would expect that by using lighter elements we would naturally move towards the right side of the map, thus increasing mobilities. However, when we go from $\mathrm{MAPbI}_{3}$ to $\mathrm{CsPbBr}_{3}$ we find that a slight increase in the phonon frequency is countered by a much heavier polar mass, and the net effect is a slight decrease in mobility, in agreement with previous calculations based on the Fröhlich model. ${ }^{39,40}$ Unlike this simplified model, direct ab-initio calculations predict a slightly larger mobility for $\mathrm{CsPbBr}_{3}$ than for $\mathrm{MAPbI}_{3}$ (see Table S3). This trend is in line with recent time-of-flight mobility measurements of $\mathrm{MAPbBr}_{3}{ }^{57,58}$ that report a higher mobility than for $\mathrm{MAPbI}_{3}$. The discrepancy between the single-phonon model of Eq. (3) and 

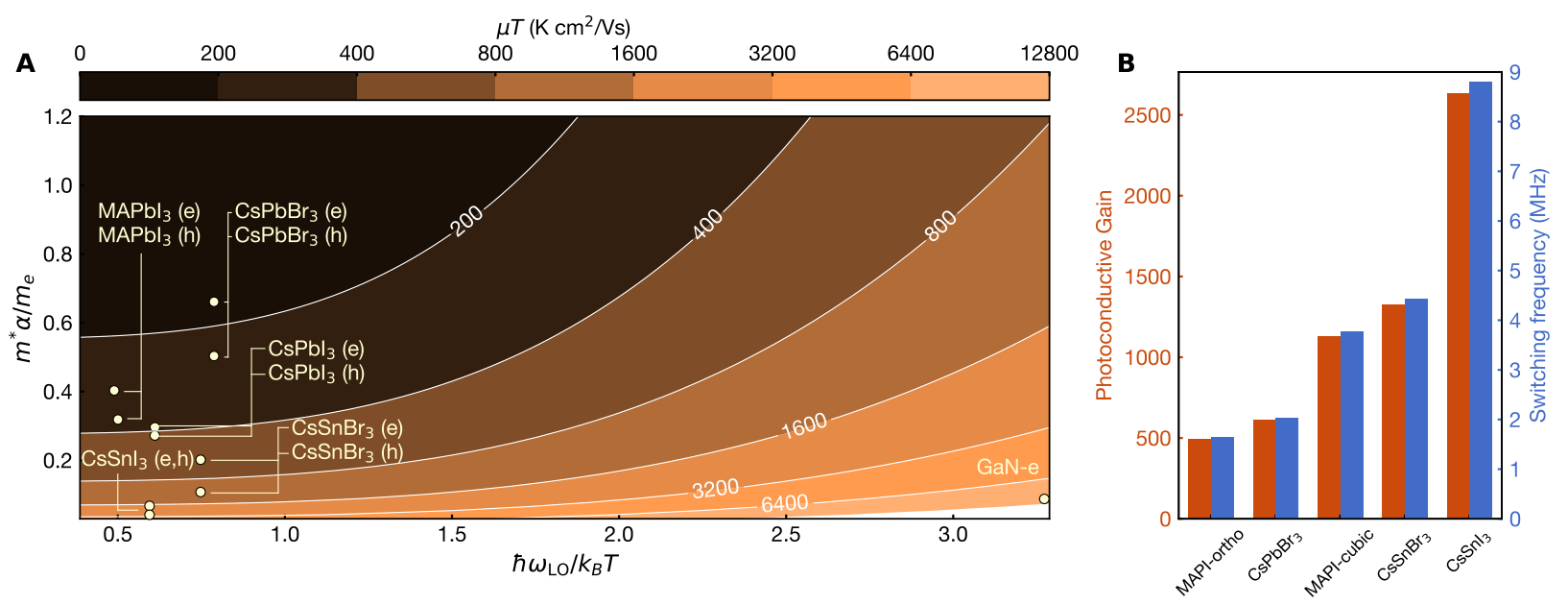

Figure 4: Mobility map of halide perovskites. (A) Calculated carrier mobility within a model including Fröhlich electron-phonon scattering of carriers in a parabolic band. The mobility depends only on the dimensionless parameters $\alpha m^{*} / m_{\mathrm{e}}$ and $\hbar \omega_{\mathrm{LO}} / k_{\mathrm{B}} T$, where $\alpha$ is the Fröhlich coupling strength, $m^{*} / m_{\mathrm{e}}$ the effective mass relative to the electron mass, and $\omega_{\mathrm{LO}}$ the frequency of the LO phonon. The details of the model are provided in Supporting Information, section 2. The predictions of the model for $\mathrm{MAPbI}_{3}, \mathrm{CsPbI}_{3}, \mathrm{CsPbBr}_{3}$,

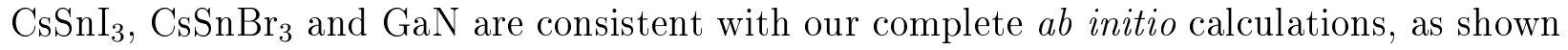
in Supporting Information, Table S3. In this table we also report the parameters $\alpha m^{*} / m_{\mathrm{e}}$ and $\hbar \omega_{\mathrm{LO}} / k_{\mathrm{B}} T$ used to locate the various compounds on the map. The horizontal coordinate of each data point corresponds to $T=300 \mathrm{~K}$, and the labels $(\mathrm{e} / \mathrm{h})$ indicate electron/hole mobilities. The map shows that the key obstacle to achieving high-carrier mobilities in halide perovskites is the very low LO phonon energy, and that one can circumvent this bottleneck by reducing the polar mass $\alpha m^{*} / m_{\mathrm{e}}$, as in the case of $\mathrm{CsSnI}_{3}$. (B) Estimated photoconductive gain (red) at $300 \mathrm{~K}$ of perovskite photodetectors and switching frequency (blue) of perovskite field-effect transistors, compared to $10^{5}$ and $0.9 \mathrm{THz}$ for GaN, respectively. ${ }^{55,56}$ We use $\mathrm{CsPbI}_{3}$ at the lattice parameter of $\mathrm{MAPbI}_{3}$ to approximate the cubic phase of the latter. The evaluation of these performance metrics is discussed in the Methods section.

the complete calculation highlights the importance of considering a multi-phonon Fröhlich coupling for an accurate description of halide perovskites, ${ }^{41}$ as can be seen in Fig. 3(a) and 3(b). In addition, the substitution of $\mathrm{Pb}$ by $\mathrm{Sn}$ appears to be very promising, as the polar mass decreases substantially, while the reduced frequency remains almost unchanged. These findings indicates that $\mathrm{Sn}$-based halide perovskites or mixed $\mathrm{Pb} / \mathrm{Sn}$ halide perovskites should be considered as primary targets in the quest for more efficient materials for halide optoelectronics.

As an illustration of the benefits of realizing high-mobility halide perovskites, we show in 
Fig. 4(b) how two figures of merit for photodetectors and thin-film transistors, the photoconductive gain and the switching frequency, vary across the map of Fig. 4(a). It is clear that the realization of stable Sn-based perovskites, mixed $\mathrm{Pb} / \mathrm{Sn}$ perovskites, and their derivatives, could lead to an order-of-magnitude increase in these performance metrics over Pb-only compounds, thereby opening entirely new opportunities in solution-processable optoelectronics.

In summary, we developed the first complete non-empirical theory of carrier mobilities in halide perovskites. We demonstrated that the maximum achievable carrier mobilities in these materials are set by the Fröhlich electron-phonon coupling associated with the stretching motion of the $\mathrm{Pb}-\mathrm{I}$ bonds. By performing a comparative analysis between halide perovskites and standard inorganic semiconductors, we established that the relatively low mobilities observed so far in these materials result from the very low frequency of the $\mathrm{Pb}-\mathrm{I}$ vibrations, which leads to significant thermal activation of phonons at room temperature. More generally, we identified a universal scaling law that underpins carrier transport in these compounds, and we proposed a mobility map to rationalize the transport properties of all halide perovskites. Our study will serve as a simple conceptual framework to understand the theoretical performance limits of these compounds, and to guide the design of new materials and devices for perovskite optoelectronics.

\section{Acknowledgement}

The research leading to these results has received funding from the Leverhulme Trust (Grant RL-2012-001), the Graphene Flagship Core2 (Grant No. 785219), and the UK EPSRC (Grant No. EP/M020517/1). The authors acknowledge the use of the University of Oxford Advanced Research Computing (ARC) facility (http://dx.doi.org/10.5281/zenodo.22558), the ARCHER UK National Supercomputing Service under the "T-Dops" project, the DECI

resource "Cartesius" based in The Netherlands at SURFsara and "Abel" based in Oslo with support from the PRACE AISBL, PRACE for awarding us access to MareNostrum at BSC- 
CNS, Spain and CSD3, UK EPSRC (Grant EP/P020259/1).

\section{Supporting Information Available}

A full description of the materials and methods used in this paper can be found in the Supporting Information and rely on Refs. ${ }^{13,16,23,24,41,52,59-70}$

The following files are available:

- Supplementary Text

- Figs. S1 to S6

- Tables S1 to S4

- References

\section{References}

(1) Green, M. A.; Ho-Baillie, A.; Snaith, H. J. The Emergence of Perovskite Solar Cells. Nat. Photon. 2014, 8, 506-514.

(2) Stranks, S.; Snaith, H. J. Metal-Halide Perovskites for Photovoltaic and Light-Emitting Devices. Nat. Nanotechnol. 2015, 10, 391-402.

(3) Kojima, A.; Teshima, K.; Shirai, Y.; Miyasaka, T. Organometal Halide Perovskites as Visible-Light Sensitizers for Photovoltaic Cells. J. Am. Chem. Soc. 2009, 131, 60506051.

(4) Lee, M. M.; Teuscher, J.; Miyasaka, T.; Murakami, T.; Snaith, H. Efficient Hybrid Solar Cells based on Meso-Superstructured Organometal Halide Perovskites. Science 2012, 338, 643-647. 
(5) Kim, H.-S.; Lee, C.-R.; Im, J.-H.; Lee, K.-B.; Moehl, T.; Marchioro, A.; Moon, S.-J.; Humphry-Baker, R.; Yum, J.-H.; Moser, J. E.; et al., Lead Iodide Perovskite Sensitized All-Solid-State Submicron Thin Film Mesoscopic Solar Cell with Efficiency Exceeding 9\%. Sci. Rep. 2012, 2, 591.

(6) Tan, Z.-K.; Moghaddam, R. S.; Lai, M. L.; Docampo, P.; Higler, R.; Deschler, F.; Price, M.; Sadhanala, A.; Pazos, L. M.; Credgington, D.; et al., Bright Light-Emitting Diodes based on Organometal Halide Perovskite. Nat. Nanotechnol. 2014, 9, 687-692.

(7) Zhu, H.; Fu, Y.; Meng, F.; Wu, X.; Gong, Z.; Ding, Q.; Gustafsson, M. V.; Trinh, M. T.; Jin, S.; Zhu, X.-Y. Lead Halide Perovskite Nanowire Lasers with Low Lasing Thresholds and High Quality Factors. Nat. Mater. 2015, 14, 636-642.

(8) Ha, S.-T.; Shen, C.; Zhang, J.; Xiong, Q. Laser Cooling of Organic-Inorganic Lead Halide Perovskites. Nat. Photon. 2015, 10, 115-121.

(9) Shrestha, S.; Fischer, R.; Matt, G. J.; Feldner, P.; Michel, T.; Osvet, A.; Levchuk, I.; Merle, B.; Golkar, S.; Chen, H., et al. High-performance Direct Conversion X-Ray Detectors based on Sintered Hybrid Lead Triiodide Perovskite Wafers. Nat. Photon. 2017, 11, 436-440.

(10) Park, S.; Chang, W. J.; Lee, C. W.; Park, S.; Ahn, H.-Y.; Nam, K. T. Photocatalytic Hydrogen Generation from Hydriodic Acid using Methylammonium Lead Iodide in Dynamic Equilibrium with Aqueous Solution. Nat. Energy 2017, 2, 16185.

(11) de Arquer, F. P. G.; Armin, A.; Meredith, P.; Sargent, E. H. Solution-Processed Semiconductors for Next-Generation Photodetectors. Nat. Rev. Mater. 2017, 2, 16100.

(12) Lin, Y.-H.; Pattanasattayavong, P.; Anthopoulos, T. D. Metal-Halide Perovskite Transistors for Printed Electronics: Challenges and Opportunities. Adv. Mater. 2017, 29, 1702838. 
(13) Senanayak, S. P.; Yang, B.; Thomas, T. H.; Giesbrecht, N.; Huang, W.; Gann, E.; Nair, B.; Goedel, K.; Guha, S.; Moya, X.; et al., Understanding Charge Transport in Lead Iodide Perovskite Thin-Film Field-Effect Transistors. Sci. Adv. 2017, 3, e160193.

(14) Yablonovitch, E. Lead Halides Join the Top Optoelectronic League. Science 2016, 351, $1401-1401$.

(15) Akinwande, D.; Petrone, N.; Hone, J. Two-dimensional Flexible Nanoelectronics. Nature Commun. 2014, 5, 5678 .

(16) Davies, C. L.; Filip, M. R.; Patel, J. B.; Crothers, T. W.; Verdi, C.; Wright, A.; Milot, R. L.; Giustino, F.; Johnston, M. B.; Herz, L. M. Bimolecular Recombination in Methylammonium Lead Triiodide Perovskite is an Inverse Absorption Process. Nat. Commun. 2018, 9, 293.

(17) Herz, L. M. Charge-Carrier Mobilities in Metal Halide Perovskites: Fundamental Mechanisms and Limits. ACS En. Lett. 2017, 2, 1539-1548.

(18) Saidaminov, M. I.; Abdelhady, A. L.; Murali, B.; Alarousu, E.; Burlakov, V. M.; Peng, W.; Dursun, I.; Wang, L.; He, Y.; Maculan, G.; et al., High-Quality Bulk Hybrid Perovskite Single Crystals within Minutes by Inverse Temperature Crystallization. Nat. Commun. 2015, 6, 7586 .

(19) Motta, C.; El-Mellouhi, F.; Sanvito, S. Charge Carrier Mobility in Hybrid Halide Perovskites. Sci. Rep. 2015, 5, 12746.

(20) Filippetti, A.; Mattoni, A.; Caddeo, C.; Saba, M. I.; Delugas, P. Low Electron-Polar Optical Phonon Scattering as a Fundamental Aspect of Carrier Mobility in Methylammonium Lead Halide $\mathrm{CH}_{3} \mathrm{NH}_{3} \mathrm{PbI}_{3}$ Perovskites. Phys. Chem. Chem. Phys. 2016, 18, $15352-15362$. 
(21) Zhao, T.; Shi, W.; Xi, J.; Wang, D.; Shuai, Z. Intrinsic and Extrinsic Charge Transport in $\mathrm{CH}_{3} \mathrm{NH}_{3} \mathrm{PbI}_{3}$ Perovskites Predicted from First-Principles. Sci. Rep. 2016, 6, 19968.

(22) Lee, J.-H.; Deng, Z.; Bristowe, N. C.; Bristowe, P. D.; Cheetham, A. K. The Competition between Mechanical Stability and Charge Carrier Mobility in MA-based Hybrid Perovskites: insight from DFT. J. Mater. Chem. C 2018, 6, 12252-12259.

(23) Poncé, S.; Margine, E. R.; Giustino, F. Towards Predictive Many-Body Calculations of Phonon-Limited Carrier Mobilities in Semiconductors. Phys. Rev. B 2018, 97, 121201.

(24) Milot, R. L.; Eperon, G. E.; Snaith, H. J.; Johnston, M. B.; Herz, L. M. TemperatureDependent Charge-Carrier Dynamics in $\mathrm{CH}_{3} \mathrm{NH}_{3} \mathrm{PbI}_{3}$ Perovskite Thin Films. Adv. Funct. Mater. 2015, 25, 6218-6227.

(25) Karakus, M.; Jensen, S. A.; D’Angelo, F.; Turchinovich, D.; Bonn, M.; Cánovas, E. Phonon-Electron Scattering Limits Free Charge Mobility in Methylammonium Lead Iodide Perovskites. J. Phys. Chem. Lett. 2015, 6, 4991-4996.

(26) Shi, D.; Adinolfi, V.; Comin, R.; Yuan, M.; Alarousu, E.; Buin, A.; Chen, Y.; Hoogland, S.; Rothenberger, A.; Katsiev, K.; et al., Low Trap-State Density and Long Carrier Diffusion in Organolead Trihalide Perovskite Single Crystals. Science 2015, $347,519-522$.

(27) Dong, Q.; Fang, Y.; Shao, Y.; Mulligan, P.; Qiu, J.; Cao, L.; Huang, J. Electron-Hole Diffusion Lengths $>175 \mu \mathrm{m}$ in Solution-Grown $\mathrm{CH}_{3} \mathrm{NH}_{3} \mathrm{PbI}_{3}$ Single Crystals. Science 2015, 347, 967-970.

(28) Shrestha, S.; Matt, G. J.; Osvet, A.; Niesner, D.; Hock, R.; Brabec, C. J. Assessing Temperature Dependence of Drift Mobility in Methylammonium Lead Iodide Perovskite Single Crystals. J. Phys. Chem. C 2018, 122, 5935-5939. 
(29) Sadhanala, A.; Kumar, A.; Pathak, S.; Rao, A.; Steiner, U.; Greenham, N. C.; Snaith, H. J.; Friend, R. H. Electroluminescence from Organometallic Lead Halide Perovskite-Conjugated Polymer Diodes. Adv. Electron. Mater. 2015, 1, 1500008-n/a, 1500008.

(30) Richter, J. M.; Abdi-Jalebi, M.; Sadhanala, A.; Tabachnyk, M.; Rivett, J. P.; PazosOutÂşn, L. M.; Gödel, K. C.; Price, M.; Deschler, F.; H., F. R. Enhancing Photoluminescence Yields in Lead Halide Perovskites by Photon Recycling and Light Outcoupling. Nat. Commun. 2016, 7, 13941.

(31) Götz, W.; Romano, L. T.; Walker, J.; Johnson, N. M.; Molnar, R. J. Hall-Effect Analysis of GaN Films Grown by Hydride Vapor Phase Epitaxy. Appl. Phys. Lett. 1998, 72, $1214-1216$.

(32) Jacoboni, C.; Canali, C.; Ottaviani, G.; Quaranta, A. A. A Review of Some Charge Transport Properties of Silicon. Solid State Electron. 1977, 20, 77-89.

(33) Stoumpos, C. C.; Malliakas, C. D.; Kanatzidis, M. G. Semiconducting Tin and Lead Iodide Perovskites with Organic Cations: Phase Transitions, High Mobilities, and NearInfrared Photoluminescent Properties. Inorg. Chem. 2013, 52, 9019-9038.

(34) Franssen, W. M. J.; van Es, S. G. D.; DerviÅ§ö̈ßlu, R.; de Wijs, G. A.; Kentgens, A. P. M. Symmetry, Dynamics, and Defects in Methylammonium Lead Halide Perovskites. The Journal of Physical Chemistry Letters 2017, 8, 61-66.

(35) Bernard, G. M.; Wasylishen, R. E.; Ratcliffe, C. I.; Terskikh, V.; Wu, Q.; Buriak, J. M.; Hauger, T. Methylammonium Cation Dynamics in Methylammonium Lead Halide Perovskites: A Solid-State NMR Perspective. The Journal of Physical Chemistry A 2018, 122, 1560-1573.

(36) Weller, M. T.; Weber, O. J.; Henry, P. F.; Di Pumpo, A. M.; Hansen, T. C. Complete 
Structure and Cation Orientation in the Perovskite Photovoltaic Methylammonium Lead Iodide between 100 and 352 K. Chem. Commun. 2015, 51, 4180-4183.

(37) Yi, H. T.; Wu, X.; Zhu, X.; Podzorov, V. Intrinsic Charge Transport across Phase Transitions in Hybrid Organo-Inorganic Perovskites. Adv. Mater. 2016, 28, 6509-6514.

(38) Chen, Y.; abd X. Wu, H. T. Y.; Haroldson, R.; Gartstein, Y. N.; Rodionov, Y. I.; Tikhonov, K. S.; Zakhidov, A.; Zhu, X. Y.; Podzorov, V. Extended Carrier Lifetimes and Diffusion in Hybrid Perovskites revealed by Hall Effect and Photoconductivity Measurements. Nature Commun. 2016, 7, 12253.

(39) Sendner, M.; Nayak, P. K.; Egger, D. A.; Beck, S.; Müller, C.; Epding, B.; Kowalsky, W.; Kronik, L.; Snaith, H. J.; Pucci, A.; et al., Optical Phonons in Methylammonium Lead Halide Perovskites and Implications for Charge Transport. Mater. Horiz. 2016, 3, 613620.

(40) Frost, J. M. Calculating Polaron Mobility in Halide Perovskites. Phys. Rev. B 2017, 96, 195202.

(41) Schlipf, M.; Poncé, S.; Giustino, F. Carrier Lifetimes and Polaronic Mass Enhancement in the Hybrid Halide Perovskite $\mathrm{CH}_{3} \mathrm{NH}_{3} \mathrm{PbI}_{3}$ from Multiphonon Fröhlich Coupling. Phys. Rev. Lett. 2018, 121, 086402.

(42) Giustino, F. Electron-Phonon Interactions from First Principles. Rev. Mod. Phys. 2017, $89,015003$.

(43) Schultz, T. D. Slow Electrons in Polar Crystals: Self-Energy, Mass, and Mobility. Phys. Rev. 1959, 116, 526-543.

(44) Ōsaka, Y. Theory of Polaron Mobility. Progress of Theoretical Physics 1961, 25, 517536. 
(45) Miyata, K.; Meggiolaro, D.; Trinh, M. T.; Joshi, P. P.; Mosconi, E.; Jones, S. C.; De Angelis, F.; Zhu, X.-Y. Large Polarons in Lead Halide Perovskites. Science Advances 2017,3 .

(46) Ambrosio, F.; Wiktor, J.; De Angelis, F.; Pasquarello, A. Origin of Low Electron-Hole Recombination Rate in Metal Halide Perovskites. Energy Environ. Sci. 2018, 11, 101105.

(47) Bretschneider, S. A.; Ivanov, I.; Wang, H. I.; Miyata, K.; Zhu, X.; Bonn, M. Quantifying Polaron Formation and Charge Carrier Cooling in Lead-Iodide Perovskites. Advanced Materials 2018, 30, 1707312.

(48) Miyata, A.; Mitioglu, A.; Plochocka, P.; Portugall, O.; Wang, J. T.-W.; Stranks, S. D.; Snaith, H.; Nicholas, R. J. Direct Measurement of the Exciton Binding Energy and Effective Masses for Charge Carriers in Organic-Inorganic Tri-Halide Perovskites. Nat. Phys. 2015, 11, 582.

(49) Saidi, W. A.; Poncé, S.; Monserrat, B. Temperature Dependence of the Energy Levels of Methylammonium Lead Iodide Perovskite from First-Principles. J. Phys. Chem. Lett. 2016, $7,5247$.

(50) Filip, M. R.; Eperon, G. E.; Snaith, H.; Giustino, F. Steric Engineering of Metal-Halide Perovskites with Tunable Optical Band Gaps. Nat. Commun. 2014, 5, 5757.

(51) Marronnier, A.; Roma, G.; Boyer-Richard, S.; Pedesseau, L.; Jancu, J.-M.; Bonnassieux, Y.; Katan, C.; Stoumpos, C. C.; Kanatzidis, M. G.; Even, J. Anharmonicity and Disorder in the Black Phases of Cesium Lead Iodide Used for Stable Inorganic Perovskite Solar Cells. ACS Nano 2018, 12, 3477.

(52) Pérez-Osorio, M. A.; Milot, R. L.; Filip, M. R.; Patel, J. B.; Herz, L. M.; Johnston, M. B.; Giustino, F. Vibrational Properties of the Organic-Inorganic Halide Per- 
ovskite $\mathrm{CH}_{3} \mathrm{NH}_{3} \mathrm{PbI}_{3}$ from Theory and Experiment: Factor Group Analysis, FirstPrinciples Calculations, and Low-Temperature Infrared Spectra. J. Phys. Chem. C 2015, 119, 25703-25718.

(53) PÃlrez-Osorio, M. A.; Lin, Q.; Phillips, R. T.; Milot, R. L.; Herz, L. M.; Johnston, M. B.; Giustino, F. Raman Spectrum of the OrganicâĂŞInorganic Halide Perovskite $\mathrm{CH}_{3} \mathrm{NH}_{3} \mathrm{PbI}_{3}$ from First Principles and High-Resolution Low-Temperature Raman Measurements. The Journal of Physical Chemistry C 2018, 122, 21703-21717.

(54) Wright, A. D.; Verdi, C.; Milot, R. L.; Eperon, G. E.; Pérez-Osorio, M. A.; Snaith, H. J.; Giustino, F.; Johnston, M. B.; Herz, L. M. Electron-Phonon Coupling in Hybrid Lead Halide Perovskites. Nat. Commun. 2016, 7, 11755, Article.

(55) Muñoz, E.; Monroy, E.; Garrido, J. A.; Izpura, I.; Sánchez, F. J.; Sánchez-García, M. A.; Calleja, E.; Beaumont, B.; Gibart, P. Photoconductor Gain Mechanisms in GaN Ultraviolet Detectors. Appl. Phys. Lett. 1997, 71, 870-872.

(56) Santoruvo, G.; Matioli, E. In-Plane-Gate GaN Transistors for High-Power RF Applications. IEEE Electr. Device L. 2017, 38, 1413-1416.

(57) Wei, Y., H. Fang; Mulligan, P.; Chuirazzi, W.; Fang, H.-H.; Wang, C.; Ecker, B. R.; Gao, Y.; Loi, M. A.; Cao, L.; Huang, J. Sensitive X-ray Detectors made of Methylammonium Lead Tribromide Perovskite Single Crystals. Nature Photonics 2016, 10, 333.

(58) Wei, H.; DeSantis, D.; Wei, W.; Deng, Y.; Guo, D.; Savenije, T. J.; Cao, L.; Huang, J. Dopant Compensation in Alloyed $\mathrm{CH}_{3} \mathrm{NH}_{3} \mathrm{PbBr}_{3-x} \mathrm{Cl}_{x}$ Perovskite single Crystals for Gamma-Ray Spectroscopy. Nature Materials 2017, 16, 826.

(59) Giannozzi, P.; Andreussi, O.; Brumme, T.; Bunau, O.; Nardelli, M. B.; Calandra, M.; Car, R.; Cavazzoni, C.; Ceresoli, D.; Cococcioni, M.; et al., Advanced Capabilities 
for Materials Modelling with Quantum ESPRESSO. Journal of Physics: Condensed Matter 2017, 29, 465901.

(60) Hamann, D. R. Optimized Norm-Conserving Vanderbilt Pseudopotentials. Phys. Rev. $B$ 2013, 88, 085117.

(61) van Setten, M.; Giantomassi, M.; Bousquet, E.; Verstraete, M.; Hamann, D.; Gonze, X.; Rignanese, G.-M. The PseudoDojo: Training and Grading a 85 Element Optimized Norm-Conserving Pseudopotential Table. Comp. Phys. Comm. 2018, 226, 39 - 54.

(62) Baikie, T.; Fang, Y.; Kadro, J. M.; Schreyer, M.; Wei, F.; Mhaisalkar, S. G.; Graetzel, M.; White, T. J. Synthesis and Crystal Chemistry of the Hybrid Perovskite $\left(\mathrm{CH}_{3} \mathrm{NH}_{3}\right) \mathrm{PbI}_{3}$ for Solid-State Sensitised Solar Cell Applications. J. Mater. Chem. A 2013, 1, 5628-5641.

(63) Vurgaftman, I.; Meyer, J. R.; Ram-Mohan, L. R. Band Parameters for III-V Compound Semiconductors and their Alloys. J. Appl. Phys. 2001, 89, 5815-5875.

(64) Mingo, N.; Stewart, D. A.; Broido, D. A.; Srivastava, D. Phonon Transmission through Defects in Carbon Nanotubes from First Principles. Phys. Rev. B 2008, 77, 033418.

(65) Marini, A.; Hogan, C.; Grüning, M.; Varsano, D. Yambo: An ab initio Tool for Excited State Calculations. Comp. Phys. Commun. 2009, 180, 1392-1403.

(66) Godby, R. W.; Needs, R. J. Metal-Insulator Transition in Kohn-Sham theory and Quasiparticle theory. Phys. Rev. Lett. 1989, 62, 1169-1172.

(67) Bruneval, F.; Gonze, X. Accurate $G W$ Self-Energies in a Plane-Wave Basis Using Only a Few Empty States: Towards Large Systems. Phys. Rev. B 2008, 78, 085125.

(68) Poncé, S.; Margine, E. R.; Verdi, C.; Giustino, F. EPW: Electron-Phonon Coupling, Transport and Superconducting Properties using Maximally Localized Wannier Functions. Comp. Phys. Commun. 2016, 209, 116-133. 
(69) Mostofi, A. A.; Yates, J. R.; Pizzi, G.; Lee, Y.-S.; Souza, I.; Vanderbilt, D.; Marzari, N. An updated Version of Wannier90: A Tool for Obtaining Maximally-Localised Wannier Functions. Comp. Phys. Commun. 2014, 185, 2309-2310.

(70) Chen, H.-W.; Sakai, N.; Jena, A. K.; Sanehira, Y.; Ikegami, M.; Ho, K.-C.; Miyasaka, T. A Switchable High-Sensitivity Photodetecting and Photovoltaic Device with Perovskite Absorber. J. Phys. Chem. Lett. 2015, 6, 1773-1779. 